\title{
ACTINOMICOSE CEREBRAL
}

\author{
OBSERVACXO ANATOMO-CLINICA E REVISAO DA LITERATURA BRASILEIRA.
}

\author{
Luciano de Souza Queiroz * \\ ANAMARLI NUCCI ** \\ J. LOPES DE FARIA **;
}

É raro o acometimento do sistema nervoso central e suas meninges por actinomicetos (Actinomyces e Nocardia) ${ }^{9}, 11,13,22$. Em recente revisão de 181 casos de actinomicose humana ${ }^{6}$, apenas cinco apresentaram lesão cerebral, sendo três com envolvimento extenso. A bibliografia nacional sobre actinomicose revela somente seis casos de comprometimento neurológico ${ }^{2}, 16$, 19, 27, todos publicados entre 1934 e 1946, não terdo o assunto recebido atenção ulteriormente.

\section{OBSERVAÇAO CLINICA}

S. S. C., 33 anos, sexo masculino, branco, brasileiro, lavrador, internado no Serviço de Emergência em 17-06-1971. Queixava-se de cefaléia difusa havia oito dias, de moderada intensidade, com periodos de exacerbacão, acompanhada de vómitos e diminuição discreta da acuidade visual. Apresentou nesse periodo dois ou três episódios de perda da consciência e convulsōes mal caracterizadas. Referia no passado teniase não tratada e crises de cefaléia. O exame clinico neurológico mostrou paciente em regular estado geral, torporoso, normotenso e afebril. Ausência de sinais de irritação meningorradicular. Estase papilar bilateral. No dimidio direito, observou-se força muscular diminuída, com manobras de Barré, Mingazzini e Raimiste positivas; reflexos miotáticos vivos; reflexos abdominais superficiais diminuidos e presença do sinal de Babinski. O paciente foi medicado com Decadron e Manitol, com discreta melhora clínica. Durante a evoluç̃o, apresentou picos febris e tosse nāo produtiva. Quatro dias após a admissão sobreveio insuficiência respiratória, sendo necessária traqueostomia. Concomitantemente instalou-se piora aguda do quadro neurológico, com perda irreversível da consciência, hipertensão arterial sistêmica $(170 / 130 \mathrm{mmHg})$ e midríase paralitica bilateral. Os exames complementares revelaram: $\mathrm{Na}+123,7 \mathrm{mEq} / \mathrm{l} ; \mathrm{K}+4,3 \mathrm{mEq} / 1$. Liquido cefalorraqueano (SOD) com pressão inicial de $32 \mathrm{cmH}^{2} \mathrm{O}$ e final de $16 \mathrm{cmH}^{2} \mathrm{O}$; retirados $2,5 \mathrm{ml}$; leucócitos $9 / \mathrm{mm}^{3}$ (linfócitos $96 \%$ e monócitos $4 \%$ ); proteínas totais $42 \mathrm{mg} \%$; glicose $98 \mathrm{mg} \%$; reaçōes de Wassermann, VDRL e Weinberg negativas; demais reações bioquímicas não executadas por insuificiência de material. Embora programados, o eletroencefalograma e angiografia cerebral não foram realizados devido às más condições gerais do paciente. Obito em 22-06-1971.

Autópsia - Adulto em regular estado de nutrição, com orificio de traqueostomia e enfisema subcutâneo. Cabeça - Dura-mater e seios venosos normais. o lobo

Trabalho da Faculdade de Ciências Médicas da Universidade Estadual de Campinas (UNICAMP): * Médico Residente do Depto. de Anatomia Patológica; ** Médica Residente de Neurologia Clínica do Depto. de Neurologia e Neurocirurgia (Chefe - Dr. Nubor O. Facure); ** Chefe do Depto. de Anatomia Patologica. 
temporal direito estava aderido à fossa craniana média e rompeu-se à retirada do encéfalo, com extravasamento de pus. Encéfalo - Peso a fresco 1.420 g. Moderado edema difuso. Leptomeninge da convexidade e da base fina e transparente, exceto em certas áreas onde estava levemente espesssada. Os cortes frontais dos hemistérios cerebrais revelaram três abscessos, assim caracterizados: a) no corte que passa pelo infundíbulo hipofisário havia um abscesso no lobo temporal direito, córtico-subcortical, arredondado, $\operatorname{com} 1,5 \mathrm{~cm}$ de diâmetro, roto durante a retirada do encéfalo; b) no corte que passa pelas bases dos pedúnculos cerebrais, um segundo abscesso no lobo parietal esquerdo, paramediano, córtico-subcortical, ovalado, medindo $2 \times 1 \mathrm{~cm}$ (Fig. 1); c) no corte que passa $3 \mathrm{~cm}$ anterior aos polos occipitais, notava-se o terceiro abscesso, na substância branca subcortical da região superior do lobo occipital esquerdo, de forma muito irregular, alongada, com cerca de $2,5 \mathrm{~cm}$ no maior diâmetro, parede constituída por tecido avermelhado e ricamente vascularizado. O hemisfério cerebral esquerdo mostrava moderado edema, com desvio da linha média. Os cortes de cerebelo e tronco cerebral nada revelaram. Cavidade torácica - Ausência de derrames nas cavidades serosas. O pulmão direito estava fortemente aderido à pleura diafragmática, rompendo-se nesse ponto durante a retirada, com extravasamento de exsudato denso, inodoro e cor de chocolate. Pulmões - O direito pesou $600 \mathrm{~g}$ e o esquerdo $400 \mathrm{~g}$. O pulmão esquerdo mostrava só enfisema e colapso parcial. O direito revelava, além disso, transformação de quase todo o lobo inferior em cavidade bronquiectásica, cheia do exsudato já descrito, onde se abria calibroso brônquio. A superficie de revestimento desta cavidade era lisa e brilhante. Os outros órgãos nada mostraram digno de nota.

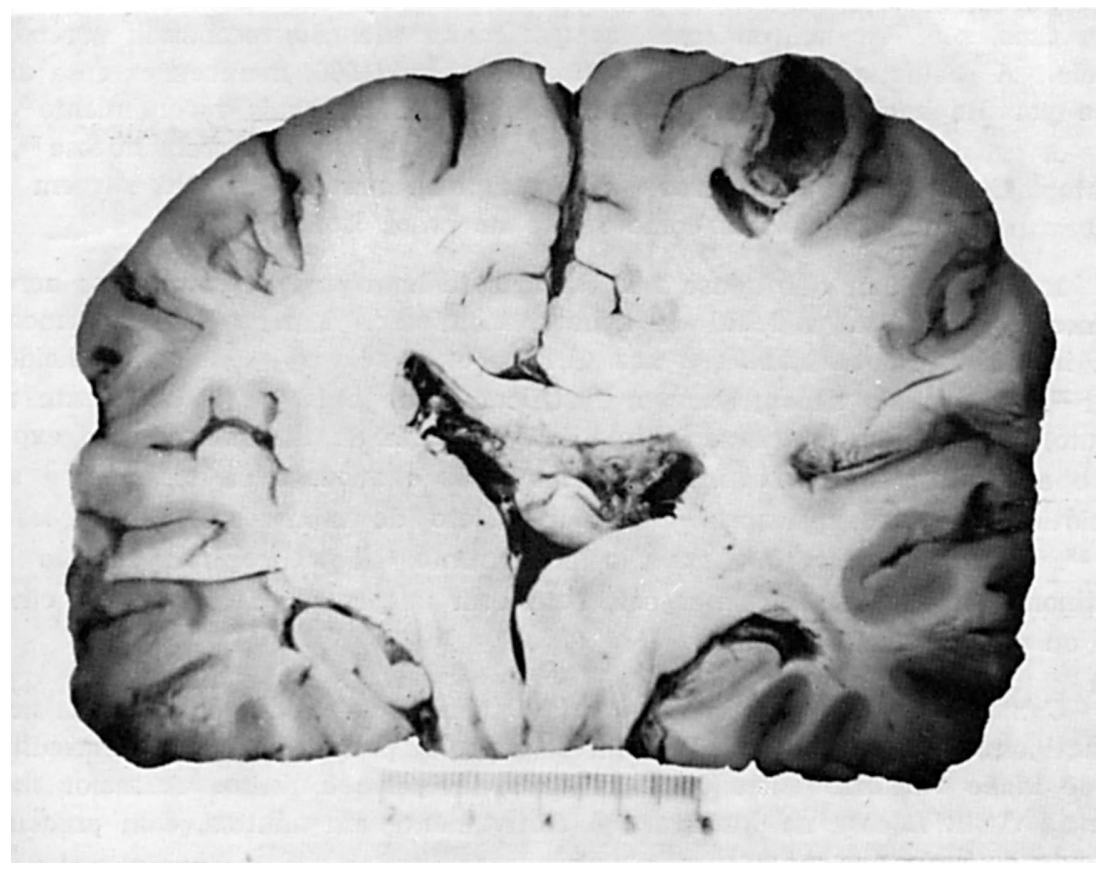

Fig. 1 - Caso S. S. C. Abscesso actinomicótico no lobo parietal esquerdo e moderado edema do centro semi-oval deste lado, causando desvio da linha média. 
Exame histopatológico. Encéfalo - O conteúdo dos abscessos referidos era constituído de leucócitos neutrófilos e apresentava algumas massas arredondadas ou irregulares, basófilas, com as caracteristicas morfológicas de grãos actinomicóticos (Fig. 2). Tais massas coravam-se intensamente em castanho escuro ou negro pela técnica argêntica de Grocott para fungos. $\mathrm{Em}$ cortes de $3 \mu$ identificaram-se nos grãos finissimos filamentos tortuosos e ramificados, de espessura inferior a $1 \mu$. A maioria dos fungos apresentava, contudo, forma cocóide ou coco-bacilar. O método de Gram (técnicas de MacCallum-Goodpasture e Brown-Brenn) evidenciou microrganismos cocóides gram-positivos só em raras colônias, e gram-negativos na maioria. A parede dos abscessos era formada por tecido de granulaçāo, com astrócitos gemistocíticos, fibroblastos, linfócitos, macrófagos e leucócitos neutrófilos. A leptomeninge adjacente mostrava moderado infiltrado inflamatório linfoplasmocitário, com macrófagos e alguns leucócitos neutrófilos. Pulmão - A parede da cavidade bronquiectásica constituia-se de epitélio cúbico simples revestindo tecido de granulação, ao qual se seguia extensa área de tecido fibroso denso neoformado, muito vascularizado e com infiltrado linfoplasmocitário focal. Alem disso, havia broncopneumonia organizante, com formação de abscessos, nos quais se observaram grãos actinomicóticos. Estes, com a técnica de Grocott, revelaram maior número de delicados filamentos que no encéfalo (Fig. 2). O carater organizante era dado por obliteração dos alvéolos pulmonares à custa de tecido fibroso rico em plasmócitos existente na luz e paredes alveolares. Os achados nos outros órgãos não foram dignos de nota.

\section{COMENTARIOS}

A criptococse $3,7,10,25$ e a blastomicose sul-americana, em suas formas nervosas, são, das neuromicoses, as que maior atenção receberam em nosso meio. A casuística nacional da blastomicose, até 1966, mereceu extensa análise por Raphael ${ }^{18}$. Outros casos foram publicados mais recentemente 5,17 , 31. A esporotricose ${ }^{23}$, a mucormicose ${ }^{29}$, a aspergilose ${ }^{30}$, a cromomicose ${ }^{24}$, a histoplasmose ${ }^{8}$, e a candidiase $^{21,32}$ do sistema nervoso central surgem na literatura brasileira apenas como relato de casos isolados.

$\mathbf{E}$ de estranhar que quase três décadas tenham passado sem novos acréscimos à casuística nacional de neuroactinomicose. Embora um incremento da incidência de actinomicose nos últimos anos seja sugerido por Scheidegger ${ }^{22}$, nada mais encontramos a justificar este ponto de vista. Neste aspecto, comporta-se a actinomicose diferentemente da candidiase, cujo explosivo aumento nas três últimas décadas, após o advento da terapêutica antibiótica e imunosupressora, tem sido objeto de numerosas publicações ${ }^{12}$, 14, 28. Brown ${ }^{6}$, em ótima revisão do assunto, não encontrou relação da actinomicose com outras infecções, nem com neoplasias, aterosclerose, cirrose ou doenças metabólicas.

Comentário da casuística nacional (Tabela 1) - Todos os casos de neuroactinomicose relatados no Brasil referem-se a pacientes do sexo masculino e de idade variando entre 15 e 52 anos, inclusive o nosso. A maior incidência desta micose na literatura é efetivamente em adultos, com predominância do sexo masculino ${ }^{11}$.

A infecção actinomicótica do sistema nervoso central provém mais freqüentemente de disseminação hematogênica a partir do foco pulmonar ${ }^{4}, 9,22$ 

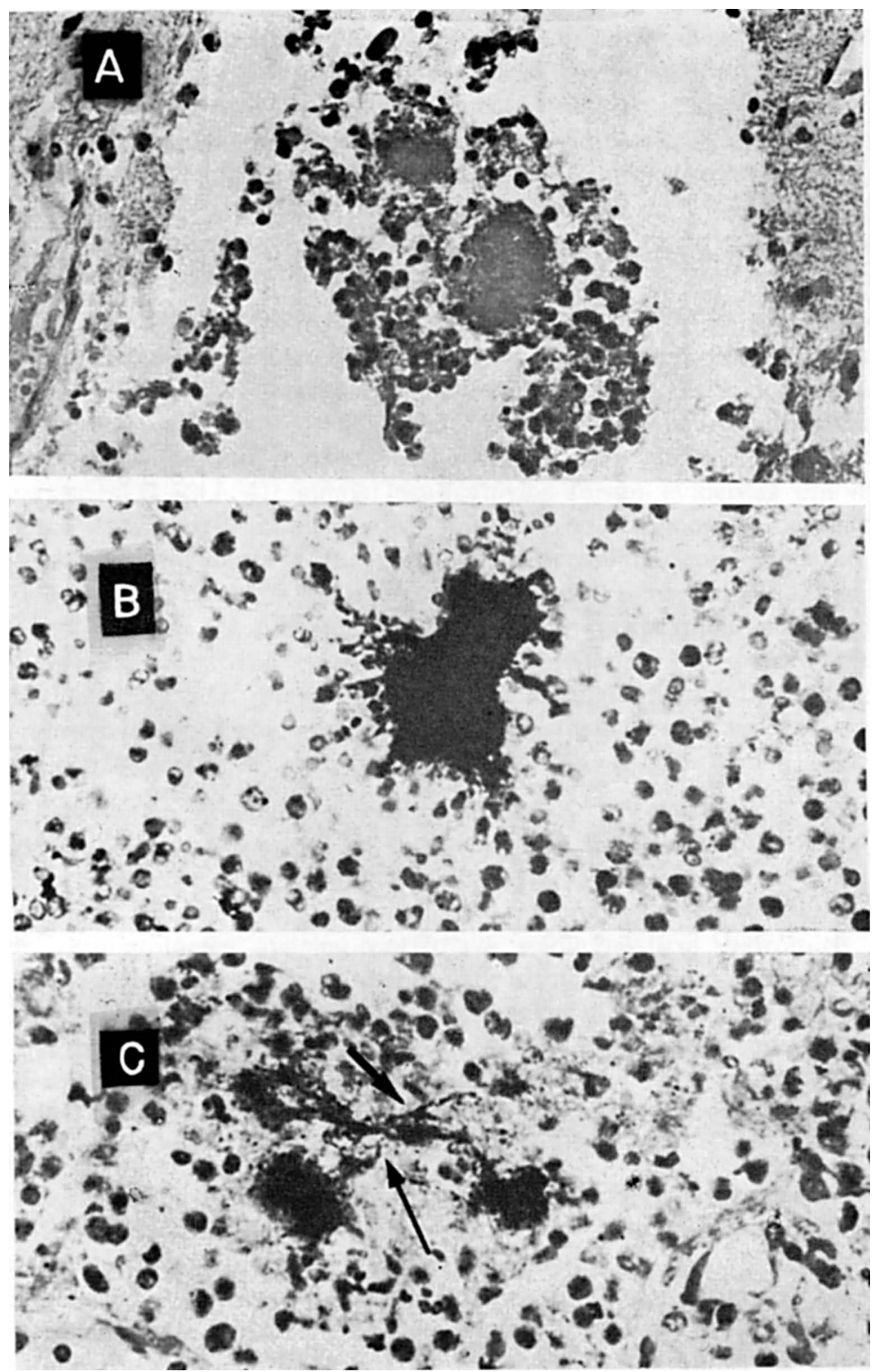

Fig. 2-Caso S. S. C. Em A, abcesso cerebral: grăos actinomicóticos entre leucócitos neutrófilos (Hematoxilina-eosina, $300 \mathrm{X}$ ); em $B$, grão actinomicótico: notar o aspecto negro compacto no centro $e$ os delicados filamentos irregulares em disposição radiada na periferia (Grocott-Gomori, $450 \mathrm{X}$ ); em C, abscesso pulmonar: notam-se dois pequenos grãos actinomicóti$\cos e$ filamentos irregulares, ramificados, por vezes com aspecto em contas de rosário (Grocott-Gomori, $500 \mathrm{X}$ ). 


\section{Caso Autor e ano}

1 Rezende, 1934

Idade

Quadro clínico

47

Exoftalmo e tumoração orbitária

E., fistulizante, dolorosa há 2 anos. Após 6 meses, sindrome meningitica.

2 Torres, 1939

41 Mal definido. Cefaléia, angủstia, dores tóraco-abdominais há 3 meses. Morte súbita.

$3 \quad$ Torres, 1939

4 Maffei, 1943

$5 \quad$ Maffei, 1943

6 Assis \& Mignone, 1946
40 Cefaléia hemicrānia E. há 7 meses. Perturbação visual há 5 meses. Edema papilar, exoftalmo E. Disfunção dos nervos II, III, V, VII à E. Obito um mês após internação.

15 Cefaléia frontal há 12 dias. Dormência no dimidio E. Fo:o:obia. Poliúria. Febre. Sonolência e sinais meningeos. Obito 10 dias após internação.

38 Cefaléia há 3 dias, antes de morte súbita.

Supuracão fistulizante ganglionar cervical há 2 anos e meio. Emagrecimento, tosse e expectoraçāo amarelada. Múltiplas fístulas torácicas. Dores difusas e radiculares dorsais. Deficit motor e sindrome piramidal de liberação frusta nos MMII. Obito 6 meses após internação.

Exames

Necrópsia

LCR - processo meningitico.

LCR - intensa hipercitose a polimorfonucleares.

LCR lombar intensa dissociação proteino-citológica.

Canal raqueano permeável.

LCR SO - albumina levemente aumentada.
Abscessos frontal e occipital à D.

Actinomicose do gânglio de Gas ser $\mathbf{E}$., ponte, polo temporal $\mathbf{E}$ Leptomeningite da convexidade à D. Trombose dos seios venosos durais.

Edema cerebral difuso. Meningite purulenta de base. Abcesso talâmico à $D$. com rotura no ventriculo lateral. Ependimite purulenta.

Edema cerebral difuso. Abcesso occipital D. Outros órgãos nada revelaram.

Intensas lesões supurativas e fistulizantes nos pulmões, pleuras, parede torácica e coluna vertebral tóraco-lombar. Paquimeningite espinal difusa sem propagacão à medula. 
ou de lesão cérvico-facial ${ }^{13}$. Só raramente o foco primário não é encontrado ${ }^{9}, 11$. No caso 1 , o foco primário era facial, e no caso 6 , cérvico-torácico, ambos com envolvimento do S.N.C. por contiguidade. No nosso caso é provável que o fungo existisse nas cavidades bronquiectásicas, daí ganhando o parênquima pulmonar e, por via hematogênica, o cérebro, formando num e noutro local os respectivos abscessos. Nos casos 2 a 5 não foi encontrada a lesão inicial, clínica ou histopatologicamente (nos casos 2 e 3 a necrópsia restringiu-se ao encéfalo).

Nos casos 2 a 5 e no caso por nós relatado, os primeiros sintomas foram neurológicos; ao contrário do que sói acontecer ${ }^{9}$, nenhum desses pacientes era portador de formas cérvico-facial, torácica ou abdominal de actinomicose que tivesse expressão clínica. Os sintomas neurológicos eram predominantemente de hipertensão intracraniana com quadro meningítico $\mathrm{cm}$ três pacientes ( 1,3 e 4$)$; sinais localizatórios foram evidentes em dois (casos 3 e 4), e no caso 6 manifestou-se síndrome de compressão medular frusta. Nosso paciente apresentava hipertensão intracraniana com moderada hemiparesia e sinais piramidais de libertação à direita, sem irritação meningorradicular. A duração da enfermidade foi de dois anos e meio nos casos 1 e 6 e variou entre três dias e sete meses nos demais. O quadro clínico da neuroactinomicose não difere sensivelmente daquele de meningites e abscessos cerebrais inespecíficos, embora a evolução mais longa favoreça o primeiro diagnóstico ${ }^{16}$.

O diagnóstico etiológico só foi estabelecido pre-mortem no caso 1, por identificação do Actinomyces no exsudato das fistulas faciais. No presente caso e nos casos 3 e 4 , o líquido cefalorraqueano, emtora com alterações inflamatórias, não permitiu o diagnóstico etiológico. No caso 6, a dissociação proteino-citológica apenas confirmava o diagnóstico sindrômico de compressão medular. Descreve-se como padrão liquórico da actinomicose a hipercitose a polimorfonucleares ou mononucleares, não superior a 1.000 células por $\mathrm{mm}^{3}$ e a normalidade ou redução da glicorraquia, que nunca atinge valores tão baixos como nas meningites bacterianas ${ }^{9,26}$. A cultura do fungo é difícil ${ }^{6}$ e, segundo Feter ${ }^{9}$, dos casos da literatura, apenas cerca de 20 foram diagnosticados por esta técnica.

Nos casos 2 a 6 , e no relatado por nós, a etiologia foi estabelecida pelo exame histopatológico do material recroscópico. Nos casos 3 e 4 , havia abscessos cerebrais e leptomeningite associadamente; o caso 6 revelava paquimeningite espinal e três casos (2, 5 e o presente) apresentaram só abscessos cerebrais. Em nosso paciente nenhum dos abscessos havia se rompido no espaço subaracnóideo ou ventricular, e a reação inflamatória da leptomeninge adjacente era do tipo crônico inespecífico, o que explica os achados de discreta hipercitose por linfomononucleares no líquido cefalorraqueano. Os abscessos actinomicóticos apresentam em geral intensa reação proliferativa e formação de tecido de granulação na parede ${ }^{9}$, 11 , sendo notável a semelhança de nosso caso com o de Bolton \& Ashenhurst *. Contudo, tal reação mais intensa é inconstante, e basicamente os abscessos actinomicóticos têm as mesmas características anátomo-patológicas dos abcessos inespecificos. A 
suspeita diagnóstica de actinomicose depende, portanto, do encontro ro pus de massas sugestivas de grãos ${ }^{13}$, que podem ser raros ${ }^{11}$. Impõe-se, assim, o emprego de colorações especiais, particularmente da técnica argêntica de Grocott para fungos, a qual demonstra com ótimo contraste os finíssimos filamentos ramificados de diâmetro inferior a $1 \mu$, característicos dos actiromicetos ${ }^{1,15}$. A impregnação diferencia o grão actinomicótico de acúmulos de bactérias ou massas de fibrina ${ }^{9}$. O método de Gram é também preconizado ${ }^{9}$, porém as hifas tendem a se corar irregularmente ${ }^{11} \mathrm{e}$, em metade dos casos revistos por Brown ${ }^{6}$, havia gram-negatividade. Tal foi, igualmente, nossa experiência nas colônias do encéfalo e concordamos com Lurie \& Duma ${ }^{15}$, que preferem a técnica de Grocott à de Gram para identificação de Nocardia asteroides. Em nosso caso, no encéfalo, as formas cocóides e coco-bacilares eram mais freqüentes, contrastando com a predominância de formas filamentosas ro pulmão. Este pleomorfismo é comumente mencionado ${ }^{6,11}$. O diagnóstico diferencial entre os gêneros Actinomyces e Nocardia não pode ser feito com segurança pelo exame histopatológico ${ }^{9}$. Aparentemente ambos podem mostrar ácido-resistência, mas só Actinomyces forma grãos em infecções viscerais; as infecções por $N$. asteroides apresentam grãos apenas na localização cutânea, mas não na visceral ${ }^{20}$.

A hipótese diagnóstica de micose deverá ser considerada em todos os casos de meningite ou de abscesso cerebral, clinicamente observados ou nos que chegam à mesa necroscópica, sob pena da incidência desses agentes continuar artificialmente reduzida em nosso meio ${ }^{16}$. Guidry ${ }^{11}$, em recente revisão, salienta que a baixa incidência de actinomicose pode estar refletindo mais a falta de diagnóstico que a escassez de casos. Recomendamos destarte a pesquisa sistemática de fungos nos casos já mencionados, utilizando-se, de preferência, a técnica argêntica de Grocott-Gomori.

\section{RESUMO}

Os autores relatam a observação anátomo-clínica de um paciente de 33 anos, com síndrome de hipertensão intracraniana e hemiparesia à direita. O liquido cefalorraqueano revelava discreta hipercitose linfomonocitária. A autópsia mostrou três abscessos cerebrais, com edema difuso mais intenso no hemisfério cerebral esquerdo, sem leptomeningite purulenta. $O$ exame histopatológico demonstrou grãos actinomicóticos nos abscessos cerebrais e pulmonares. A literatura brasileira sobre neuroactinomicose é analisada, com encontro de seis casos, publicados entre 1934 e 1946. São discutidos aspectos clínicos e anátomo-patológicos relevantes dos casos nacionais.

\section{SUMMARY}

Cerebral actinomycosis: case report and review of the Brazilian literature

A case of central nervous system actinomycosis is reported. A 33-yearold male complained of headache, vomiting and blurred vision lasting for 
eight days prior to admission. On examination, a right hemiparesis, as well an intracranial hypertension were detected. The cerebrospinal fluid showed mild lymphomononuclear hypercytosis. Necropsy disclosed three abscess in the cerebral hemispheres, in addition to moderate cerebral edema on the left side but without purulent leptomeningitis. Actinomyces filaments and granules were demonstrated in the cerebral and lung abscesses. The Brazilian literature on actinomycosis is reviewed and six published cases with nervous system involvement were found. Relevant clinical and anatomical aspects of the cases and of the present one are discussed.

\section{REFERENCIAS}

1. ANTHONY, P. P. - A guide to the histological identification of fungi in tissues. J. Clin. Pathol. 26:828, 1973.

2. ASSIS, J. L. \& MIGNONE, C. - Actinomicose toracovertebral com paquimeningite e compressão medular. Arq. Neuro-Psiquiat. (São Paulo) 4:21, 1946.

3. BECKER, P. F. L. - Criptococose. Apresentação de um caso, associado a granulomas esquistossomóticos. Revisão e estudo dos casos nacionais. Rev. Goiana Med. 6:207, 1960.

4. BOLTON, C. F. \& ASHENHURST, E. M. - Actinomycosis of the brain. Case report and review of the literature. Canad. Med. Assoc. J. 90:922, 1964.

5. BRAGA, F. M. \& OKAMURA, M. - Blastomicose medular. (Apresentação de um caso cirúrgico). Seara Med. Neurocir. 1:435, 1973.

6. BROWN, J. R. - Human actinomycosis. A study of 181 subjects. Human Pathol. 4:319, 1973.

7. Carvalho, R. A. D.; TIlbery, C. P. \& LANCellotTi, C. L. P. - Criptococose encefálica, forma tumoral. Relato de dois casos. Neurobiol. (Recife) $36: 261,1973$.

8. DUARTE, E. - Histoplasmose. Mem. Inst. Oswaldo Cruz (Rio de Janeiro) 43:457, 1945 .

9. FETTER, B. F.; KLINTWORTHH, G. K. \& HENDRY, W. S. - Mycoses of the Central Nervous System. Williams \& Wilkins, Baltimore, p. 13-24, 1967.

10. GIORGI, D. R.; REIS, J. B.; BEI, A. \& REIS FILHO, J. B. - Criptococose do sistema nervoso central. Experiência atual do Serviço de Neurologia da Escola Paulista de Medicina. Arq. Neuro-Psiquiat. (São Paulo) 32:77, 1974.

11. GUIDRY, D. J. - Actinomycosis. In Handbuch der speziellen pathologischen Anatomie und Histologie, Band III, Teil 5: The Pathologic Anatomy of Mycoses (R. D. BAKER, ed.). Springer, Berlin Göttingen Heidelberg, p. 1019-1058, 1971.

12. ISSEL, W. - Úber den Befall des Zentralnervensystems mit Candida albicans. Ärztl. Forsch. 25:34, 1971.

13. LACAZ, C. S. - Manual de Micologia Médica. 3.e ed., Livraria Atheneu S.A., Rio de Janeiro, São Paulo, 1960.

14. LOURIA, D. B.; STIFF, D. P. \& BENNETT, B. - Disseminated moniliasis in the adult. Medicine (Baltimore) 41:307, 1962 .

15. LURIE, H. I. \& DUMA, R. J. - Opportunistic infections of the lungs. Human Pathol. 1:233, 1970 .

16. MAFFEI, W. E. - Micoses do sistema nervoso. Anais Fac. Med. Univ. S. Paulo 19:297, 1943.

17. PIMENTA, A. M.; MARQUES, J. S. \& SETTANI, F. A. P. - Blastomicose cerebral, forma tumoral. Apresentação de 7 casos. Seara Med. Neurocir. 1:73, 1972.

18. RAPHAEL, A. - Localização nervosa da blastomicose sul-americana. Arq. Neuro-Psiquiat. (São Paulo) 24:69, 1966. 
19. REZENDE, M. O. - Sobre um caso de actinomicose da região orbitária esquerda, com invasão endocraniana. Micetoma e grãos branco-amarelados, Discomices brasiliensis (Lindenberg, 1909). Rev. Otolaringol. (São Paulo) 2:287, 1934.

20. ROBBOY, S. J. \& VICKERY Jr., A. L. — Tinctorial and morphologic properties distinguishing actinomycosis and nocardiosis. New Engl. J. Med. 282:593, 1970.

21. ROCHA, J. M. - Candidiase, doença geral. Bol. Inst. Puer. Univ. Brasil $22: 143,1965$.

22. SCHEIDEGGER, S. - Pilzerkrankungen. In Handbuch der speziellen pathologischen Anatomie Histologie (O. LUBARSCH, F. HENKE \& R. ROSSLE, eds.), Band XIII, Teil 2/A, Springer, Berlin Göttingen Heidelberg, p. 1183-1188, 1958.

23. SPINA-FRANCA NETTO, A.; ANGUINAH, A.; SOUZA, H. S. A. de; FAVA NETTO, C. \& PEREZ-GUISASOLA - Leptomeningite esporotricótica. Arq. Neuro-Psiquiat. (São Paulo) 28:73, 1970.

24. SPINA-FRANÇA NETTO, A.; BRITTO, T. \& ALMEIDA, F. P. - Cromomicose do sistema nervoso. Estudo anátomo-clínico de um caso. Arq. Neuro-Psiquiat. (São Paulo) 11:265, 1953.

25. SPINA-FRANCA NETTO, A. \& SILVA, J. B. - Diagnóstico e tratamento da criptococose do sistema nervoso central. Arq. Neuro-Psiquiat. (São Paulo) 26: 115, 1968.

26. TENG, P. - Actinomycotic cerebral abscesses - a report of two cases with recovery. J.A.M.A. 175:807, 1961.

27. TORRES, D. M. G. - Estudo sobre as lesōes histopatológicas em dois casos de actinomicose do S.N.C. Anais Fac. Med. Univ. S. Paulo 15:235, 1939.

28. WINNER, H. I. \& HURLEY, R. - Candida albicans. J, \& A. Churchill Ltd., London, p. 140-151, 1964.

29. WITTIG, E. O.; CAT, I.; ABDALA, H. \& KASTING, G. - Meningencefalite a mucormicose. Relato de um caso. Arq. Neuro-Psiquiat. (São Paulo) 31:151, 1973.

30. WITTIG, E. O.; CAT, I. \& KASTING, G. - Meningencefalite a aspergillus. Arq. Neuro-Psiquiat. (São Paulo) 31:220, 1973.

31. WitTIG, E. O.; KAStiNG, G. \& LEAL, R. - Neuroblastomicose. Registro de três casos. Arq. Neuro-Psiquiat. (São Paulo) 26:73, 1968.

32. WITTIG, E. O.; LOPES, R. W. \& KASTING, G. - Meningencefalite a candida. Relato de um caso. Arq. Neuro-Psiquiat. (São Paulo) 31:146, 1973.

Depto. de Patologia - Faculdade de Ciências Médicas, UNICAMP - Caixa Postal 1170 - 13100 Campinas, SP - Brasil. 